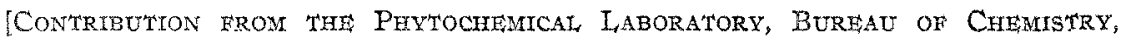
U. S. DEPARTMENT OF AGRICULTURE.]

\title{
THE ODOROUS CONSTITUENTS OF APPLES. EMANATION OF ACETALDEHYDE FROM THE RIPE FRUIT.
}

By Frederick B. Power and Victor K. Chesnut.

Received May 24, 1920.

The fact that many ripe fruits possess characteristic odors is well known, but it is somewhat remarkable that so little information has been recorded respecting the chemical nature of the substances to which these odorous properties are due. This deficiency of knowledge may, however, be attributed to the extremely small proportion in which such constituents are usually present, to their more or less complex character, and to the consequent difficulties attending their separation or identification. The respective odorous substances have commonly been regarded as esters, and a number of artificially prepared compounds of this class have indeed been given names which indicate their resemblance in odor to certain fruits, such as "apple oil" for amyl valerate, "pear oil" for amyl acetate, and "pineapple oil" for ethyl butyrate. The so-called artificial fruit essences are, however, usually composed of a mixture of substances, without any consideration of their actual occurrence in the fruits whose flavors they are supposed to represent. An actual identification of the principal flavoring constituent of an edible fruit appears to have been first accomplished by Clemens Kleber, ${ }^{1}$ who has shown by exact analytical data that ripe bananes contain anyl acetate, and, so far as known to t1s, this has remained to the present time the only recorded instance in which the chemical identity of a natural fruit flavor has been determined.

Although amyl valerate is clesignated in most chemical text-books as "apple oil," there appears to be no evidence that this compound has ever actually been found in the apple. So far as can be ascertained from a survey of chemical literature the only published references to the odorous constituents of apples are contained in 2 brief communications by $\mathrm{C}$. Thomae," who has noted that when fresh apple parings are mixed with water and distilled in a current of steann a little solid substance passes over, while occasionally some oil drops are formed which soon for the most part solidify. By extracting the distillate with ether a solid mass was obtained, which on the addition of a little alcohol became crystalline. After separating the crystals by filtration the liquid yielded a yellowish oil which had the odor of apples. Other experiments were made by extracting fresh apple parings with ether, whereby wax-like substances and some drops of yellowish oil were obtained. The author does not state the quantity of material employed, and no product of the investigation was further characterized or identified.

1 Am. Perfumer, 7, 235 (1913).

2 J. prakt. Chem.84, 247 (IgrI);87, 42 (1913). 


\section{Experimental.}

The material employed in the present research was generously provided by Mr. L. C. Corbett, in charge of horticultural and pomological investigations of the Bureau of Plant Industry, and consisted of fruit grown on the experimental farm at Arlington, Va. To him and all others of the above-mentioned Bureau of the Department of Agriculture who so kindly placed at our disposal the facilities for preparing and distilling the material our warmest thanks may here be expressed.

\section{Part I. Examination of Ben Davis Apples.}

The variety of apple first examined was that known as the Ben Davis, as this was available in considerable quantity, and as the odorous constituents appear to be contained chiefly in the outer skin or rind of the fruit, only the parings were employed. The apples had been stored in a cellar without refrigeration, and at the time of distillation, in the latter part of October, had developed considerable odor. They were pared by means of a machine, which was so adjusted as to completely remove the rind with only a very small portion of the white substance of the apple, and without removing the core, so that any admixture of seeds was carefully excluded. In order to avoid any possibility of fermentation or loss of volatile substances the fresh parings were brought immediately into a still, and, without any further addition of water, subjected to distillation. in a current of steam. The total amount of parings employed from the above-mentioned variety of apples was about $16 \mathrm{r} \mathrm{kg}$., representing approximately $805 \mathrm{~kg}$. of the entire fruit. The amount of aqueous distillate collected was 163 liters. This distillate was somewhat opalescent, and the first portions separated some oily drops, but on keeping for a few days it became perfectly clear, with the separation of a little solid material on the sides of the glass vessels containing it. The liquid possessed in a high degree the pleasant, characteristic odor of fresh, ripe apples, and even the first portions of the distillate were perfectly neutral to test-paper. After decanting the aqueous liquid, the above-mentioned solid material was dissolved by means of ether, the ethereal solution dried with anhydrous sodium sulfate, and the solvent evaporated. A small amount ( $0.04 \mathrm{~g}$.) of a colorless, flocculent substance was thus obtained, which was soluble in hot alcohol, but separated for the most part on cooling. When collected on a filter, and dried on a porous plate, it formed thin, satiny laminae, which were not altered by contact with either conc. nitric or sulfuric acid. The substance melted quite sharply at $63^{\circ}$ and was probably slightly impure triacontane, $\mathrm{C}_{30} \mathrm{H}_{62}$, which is stated to melt at $65.6^{\circ}$.

Concentration of the Distillate. Identification of Furfural and Acetaldehyde.

A large portion of the original aqueous distillate, amounting to 106 liters and representing ro $\mathrm{kg}$. of apple parings, was repeatedly cohobated 
in a current of steam, whereby the odorous substances were eventually concentrated in a volume of about one liter, the nearly odorless liquids remaining from this treatment being reserved for further examination. The concentrated distillate, which was practically netitral and contained some globules of colorless oil floating on the surface, was first subjected to some special tests. It gave no coloration with ferric chloride, indicating the absence of phenolic substances, but rapidly reduced an ammoniacal solution of silver oxide in the cold, producing a metallic mirror, and also gave an immediate red coloration with sensitized Schiff's reagent, ${ }^{1}$ which was evidence of the presence of an aldehyde. A few drops of the liquid gave with aniline and hydrochloric acid the bright red coloration characteristic of furfural, the latter having doubtless been produced in the process of distillation. When tested for formaldehyde by means of phenylhydrazine hydrochloride ${ }^{2}$ a perfectly negative result was obtained. On the other hand the distillate gave the specific reaction for acetaldehyde, which consists in adding to a little of the liquid in a test-tube one or two drops of a solution of dimethylamine $(33 \%)$ and subsequently about 5 drops of a freshly prepared $1 \%$ aqueous solution of sodium nitroprusside, when a beatutiful indigo-blue color is produced, soon changing to brown or yellow. Rimini ${ }^{3}$ has shown that the reaction for acetaldehyde as obtained by Simon ${ }^{4}$ with trimethylamine was due to the presence in the latter of dimethylamine, and that it is given by all secondary bases of the aliphatic series or by ring compounds which have completely lost their aromatic character by hydrogenation. We have found that the abovementioned blue coloration is readily obtained with a solution of one part by weight of acetaldehyde in 10,000 parts of water, but that it is not produced by formaldehyde, the higher fatty aldehydes, such as $n$-heptylic aldehyde, or by aliphatic terpene aldehydes, such as citral, which gives only a reddish color. Further confirmation of the presence of acetaldehyde, and evidence of its occurrence as an emanation from ripe apples, will subsequently be described.

\section{Hydrolysis of the Esters Contained in the Concentrated Distillate. Identification of Amyl Alcohol and Formic and Caprylic Acids.}

The previously mentioned concentrated distillate, amounting to about one liter, was brought into a flask provided with an inverted condenser, $25 \mathrm{~g}$. of pure sodium hydroxide added, and the mixture kept in active ebullition for 2 hours. During this operation the liquid acquired a deep yellow color and a small amount of material separated, which evidently

1 Mulliken, "Iclentification of Pure Organic Compounds," Vol. I, p. 15 (I905).

"Z. Nahr. Genussm, 5, 353 (I902) and J. Assoc. Official Agr. Chemists, Methods of Analysis, IgI6, p. I47.

${ }^{3}$ Annali Farmacoterapia e Ch., 1898, p. 249; Chem. Centr., r898, Bd. Ir, p. 277.

4 Compr. rend., I25, rios (1897); J. Chem. Soc., 74, II, 3I5 (I898). 
consisted of the so-called aldehyde resin. After cooling, the contents of the flask were distilled in a current of steam, and about $300 \mathrm{cc}$. of liquid was collected. This liquid, which gave no reaction for aldehyde, possessed a pleasant odor, ${ }^{1}$ and after a short time an appreciable amount of yellowish, oily drops separated on the surface. It was extracted 5 times with pure, aldehyde-free ether, and the neutral aqueous liquid then reserved in order to test for the presence of methyl alcohol, as subsequently described.

The above-mentioned ethereal liquids were united, dried with anhydrous sodium sulfate, and the greater part of the ether removed by distillation, the residual liquid being then brought into a small, tared flask and allowed to evaporate spontaneously until the odor of ether had disappeared. A yellowish, oily liquid was thus obtained, which had a distinct odor of anyl alcohol, and produced the characteristic irritating effect of the latter on the throat when inhaled. The amount of this liquid was $0.7063 \mathrm{~g}$., which represented $0.00065 \%$ of the weight of apple parings employed. It was brought into a strong flask provided with a tightly-fitting glass stopper, $25 \mathrm{cc}$. of a chromic acid mixture ${ }^{2}$ added, and the whole heated for I 5 minutes on a water bath at a temperature of about $90^{\circ}$ with occasional agitation. The flask was then cooled, the contents diluted with water, transferred to a separatory funnel, and the acid liquid extracted 5 times with pure, aldehyde-free ether. The united ethereal liquids, after being washed with a little water, were extracted 4 times successively with $25 \mathrm{cc}$. of a $5 \%$ solution of barium hydroxide. The total alkaline liquid was treated with carbon dioxide for the removal of the excess of baryta, and, after filtration, the neutral liquid was concentrated to a small volume. On the addition of silver nitrate a small amount of a nearly colorless precipitate was obtained, which was collected, washed, atid dried on a porous tile over sulfuric acid.

Subs., 0.0358: Ag, 0.0I85.

Calc. for $\mathrm{AgC}_{5} \mathrm{H}_{9} \mathrm{O}_{2}: \mathrm{Ag}, 5 \mathrm{I} .7$. Found: $5 \mathrm{x} .6$.

The acid obtained by the oxidation of the above-described hydrolytic product was thus found to be valeric acid, and its formation established the presence of amyl alcohol in the apple distillate.

After extracting the above-mentioned ethereal liquid with baryta it was washed with a little water, dried with anhydrous sodium sulfate, and the ether allowed to evaporate. A small amount of an oily liquid was thus obtained, which was mixed with water and distilled in a current of

1. It is known that acetaldehyde when heated with a caustic alkali is chiefly converted into the so-called aldehyde resin, and that small amounts of formic and acetic acids are also produced, together with some odorous substances which may be extracted from the distilled liquid by means of ether. The formation of these products has been duly considered when necessary in the present investigation in order to exclude any incorrect deductions.

J. prakt. Chem., 45, 599 (1892). 
steam. The first portion of the distillate separated some drops of a fragrant oil, and, assuming it to be an ester, the entire distillate was subjected to hydrolysis with sodium hydroxide and further examined in the manner previously described. The oily product was thus found to consist of amyl valerate, which had been formed together with free valeric acid by the oxidation of the amyl alcohol.

The yellow, strongly alkaline liquid remaining from the hydrolysis and subsequent distillation of the concentrated apple distillate was filtered in order to remove the small amount of aldehyde resin, which, when washed and dried, amounted to $0.63 \mathrm{~g}$. The liquid was then acidified with sulfuric acid and distilled in a current of steam. About 2 liters of clistillate was collected, the first portions of which had a pronounced odor and acid reaction, and separated a few oily drops. The entire distillate was made alkaline with barium hydroxide, concentrated, filtered, and the excess of baryta removed by carbon dioxide. The clear, filtered solution of barium salt was found to contain a considerable amount of formate, which was removed by treatment in the cold with a $4 \%$ solution of potassium permanganate, a current of carbon dioxide being meantwile passed through the liquid. After removing the slight excess of permanganate by a gentle heat, and filtering to separate the manganese dioxide, the colorless, neutral liquid was concentrated. It then gave with silver nitrate a small amount of a nearly white precipitate, which was collected, washed, and dried on a porous tile over suffuric acid.

Subs, , 0.0449: Ag, 0.0192.

Calc. for $\mathrm{AgC}_{8} \mathrm{H}_{15} \mathrm{O}_{2}: \mathrm{Ag}$, 43.o. Found: 42.8 .

This result is thus seen to indicate the presence of caprylic acid, of which further evidence was subsequently obtained. The filtrate from this salt gave no further precipitate with silver nitrate, and no additional amount of a definite silver salt could be obtained from it by concentration. The residue from its evaporation, however, when heated with absolute alcohol and a little conc. sulfuric acid, developed a fruity odor, which was evidence of the presence of small amounts of the lower fatty acids. In addition to the above-mentioned formic and caprylic acids, as obtained in the process of hydrolysis, the identification of other acids in the original distillate will subsequently be described.

It was previously noted that after hydrolysis of the concentrated apple distillate the subsequent distillate from the alkaline liquid was first extracted with ether and then reserved in order to test it for methyl alcohol. This was conducted by first saturating the liquid with common salt, filtering, extracting with light petroleum (b. p. $30^{-}-55^{\circ}$ ), and distilling the salt solution. After repeated concentration of this distillate it was tested according to the method of Denigès, ${ }^{1}$ as elaborated by von Fellen-

¿ "Recherche de traces de méthanal en présence d'éthanal par la fuchsine bisulfitée," Compt. rend., 150, 529 (I910). 
berg, ${ }^{1}$ which depends upon the oxidation of methyl alcohol in strongly acid solution to formaldehyde. A reaction was obtained which showed quite definitely the presence of traces of methyl alcohol, and it was ascertained by control experiments that this was not contained in the ether which had previously been employed for extracting the liquid examined. This result was, moreover, confirmed by some special experiments, to be described later, which completely established the occurrence of small amounts of both methyl and ethyl alcohols in apple parings.

\section{Examination of the Aqueous Liquids Remaining from the Concentration of the Original Distillate. Identification of Formic, Acetic, Caproic and Caprylic Acids.}

The large quantity of nearly odorless liquid remaining from the cohobation of 106 liters of the original distillate, together with that obtained by the subsequent concentration of 57 liters of distillate, as later described, was made alkaline with sodium hydroxide and evaporated to a volume of about one liter. It was then acidified with sulfuric acid and distilled in a current of steam. About 5 liters of distillate was collected, the first portions of which were turbid, had a strongly acid reaction, and separated some oily drops. The entire amount of distillate, which had an odor resembling that of the higher fatty acids, was made alkaline with barium hydroxide and evaporated to a small volume. After filtration the excess of baryta was removed by carbon dioxide, and the liquid again filtered and concentrated. It was found to contain a large amount of formic acid, which was removed by treatment with a cold $4 \%$ solution of potassium permanganate, a current of carbon dioxide being meanwhile passed through the liquid. After the separation of the manganese dioxide by gently heating and filtering, the colorless, neutral solution was concentrated. By the subsequent addition of silver nitrate a number of silver salts were obtained, which gave the following figures on analysis.

Fraction I. Subs., 0.0139: $\mathrm{Ag}, 0.0059$.

Calc. for $\mathrm{AgC}_{8} \mathrm{H}_{15} \mathrm{O}_{2}: \mathrm{Ag}, 43.0$. Found: 42.4 .

This result indicates the presence of a small amount of caprylic acid, which had been found in larger proportion in the products obtained from the concentrated distillate after hydrolysis.

Fraction If. Subs., $0.0370: \mathrm{Ag}, 0.0177$

III. Subs, 0.0457: Ag, 0.0223. Subs, 0.0394: Ag, 0.0r92.

Calc. for $\mathrm{AgC}_{6} \mathrm{H}_{11} \mathrm{O}_{2}: \mathrm{Ag}, 48.4$. Found: II, 4.7.8; III, 48.8, 48.7.

These fractions evidently represented salts of caproic acid, and they all developed the characteristic fatty odor on ignition.

The filtrate from the preceding fractions yielded successively the following additional silver salts.

I Biochem. Z., 85, 45 (1918). 


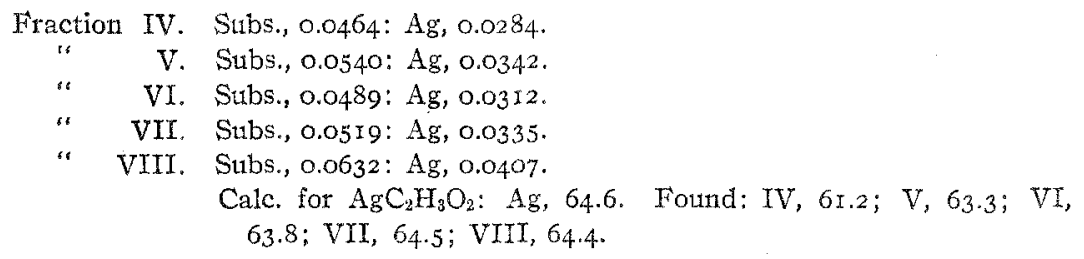

It may be concluded from the above results that the volatile acids of the Ben Davis apple are chiefly formic, acetic and caproic acids, with a very small amount of caprylic acid. There was no evidence of the presence of valeric acid.

\section{Extraction and Characters of the Essential Oil.}

In order to ascertain the general characters of the combined volatile constituents of the apple a portion of the original distillate amounting to 57 liters, which represented $54 \mathrm{~kg}$. of the parings, was repeatedly cohobated in a current of steam until the odorous substances were finally contained in a volume of about 8 liters. The practically odorless liquids remaining in the large distilling flasks were mixed with those obtained by the cohobation of a larger quantity of the original distillate, and the whole examined as already described.

The above-mentioned concentrated distillate was extracted 4 times successively with specially purified ether, which was free from aldehyde. The united ethereal liquids were dried with anhydrous sodium sulfate, and the ether removed for the most part by distillation, after which the liquid was brought into a small, tared fask, and the remainder of the ether allowed to evaporate spontaneously. The amount of volatile product so obtained was $\mathrm{I.905} \mathrm{g}$, thus representing $0.0035 \%$ of the apple parings or about $0.0007 \%$ of the entire frut.

The essential oil was a yellow, somewhat viscid liquid, which gradually became darker on keeping, and possessed in a high degree the pleasant, characteristic odor of fresh apples. When cooled slightly below the ordinary room temperature it formed a soft, concrete mass, due to the separation of minute, acicular crystals, which evidently consisted of the previously mentioned paraffin hydrocarbon. A few drops of the oil, when shaken with a little water, gave all the above-thoted reactions for acetaldehyde, and it also gave a decided reaction for furfural with aniline and hydrochloric acid.

The concentrated aqueous distillate which had been extracted with ether for the removal of the essential oil was made alkaline with sodium hydroxide and evaporated. It was then acidified with sulfuric acid and distilled in a current of steam. The faintly acid distillate was made alkaline with baryta, concentrated, and the excess of baryta removed by carbon dioxide. After filtration and concentration the liquid was found 
to contain an appreciable amount of formate, which was removed by oxidation with a $4 \%$ solution of potassium permanganate in a current of carbon dioxide. The small amount of colorless solution eventually obtained gave on the addition of silver nitrate only a slight precipitate, which was too small for examination, but on concentrating the filtrate therefrom a very small amount of a silver salt was deposited, which was collected, washed with water, dried and analyzed.

Subs., 0.0156: $\mathrm{Ag}$, 0.010x.

Calc. for $\mathrm{AgC}_{2} \mathrm{H}_{8} \mathrm{O}_{2}: \mathrm{Ag}, 64.6$. Found: 64.7 .

The distillate from which the essential oil had been removed was thus found to contain only formic and acetic acids.

\section{Part II. Examination of Springdale Apples. Occurrence of Traces of Methyl and Ethyl Alcohols.}

It was noted in connection with the examination of Betn Davis apples that traces of methyl alcohol were found in the distillate. In order to confirm this observation it was deemed desirable to conduct a special experiment in which there could be no possibility of error, although the purified ether used for the previous extraction of the distillate had been carefully tested and found to be free from methyl alcohol. The variety of apples available for this purpose was that known as Springdale, and they were examined in the latter part of January.

A quantity of fresh parings from the apple, amounting to about 7.5 $\mathrm{kg}$., was immediately distilled in large glass flasks by means of a current of steam, and about 5 liters of distillate were collected. The first portions of the distiliate, which had a fragrant odor and contained some drops of oil, had a faintly acid reaction and responded to the tests for acetaldehyde. The entire amount of distilled liquid was repeatedly cohobated until the odorous or more volatile constituents were finally contained in a voitune of about $500 \mathrm{cc}$. To this liquid, contained in a flask provided with an inverted condenser, Io g. of sodium hydroxide was added, and the mixture kept in active ebullition for 2 hours. It had then acquired a deep yellow color with the separation of a small amount of aldehyde resin. After cooling, the alkaline mixture was distilled, and $20 \mathrm{cc}$. of distillate collected. This liquid, which had a pleasant odor, was saturated with common salt, and then extracted with light petroletum for the purpose of removing any higher alcohols. The salt solution was subsequently distilled, and this distillate subjected to further repeated distillations until finally I.5 cc. was collected, the receiver being cooled with ice. A small portion (o.I cc.) of this liquid when oxidized with potassium permanganate in strongly acid solution developed a distinct odor of formaldehyde, and when further treated according to the method of Denigès ${ }^{1}$ a decided reaction for this

1 Loc, cit. 
aldehyde was obtained. As the last-mentioned substance was not contained in the original apple distillate, the presence of mintute amounts of methyl alcohol was definitely established. It could naturally not be determined whether this alcohol was contained in the original distillate as such or in the form of an ester, and it may possibly have been produced during the distillation of the apple parings by the hydrolysis of a methyl ester of pectin. ${ }^{1}$

The remainder of the above-mentioned $1.5 \mathrm{cc}$. of concentrated distillate was tested for the presence of ethyl alcohol by means of the iodoform reaction, when distinct crystals of this substance were obtained.

The occurrence of ethyl alcohol in fruits and other parts of plants which have not undergone fermentation has previously been recorded by several observers. ${ }^{2}$ Moreover, Gutzeit, ${ }^{3}$ by the distillation of several umbelliferous fruits, obtained both methyl and ethyl alcohols, which may have existed in the form of esters, and methyl alcohol or its esters was likewise found by Lieben ${ }^{4}$ in the products of distillation of meadow grass and the leaves of trees. It has, furthermore been noted by $A$. Gautier that he obtained $0.8 \mathrm{~g}$. of ethyl alcohol from $300 \mathrm{~g}$. of so-called pommes gelees, an expression which refers to a condition of some apples known as "water-core," but the fruit employed by us was of perfectly normal appearance.

\section{Other Constituents of Springdale Apples.}

A11 the aqueous liquids remaining from the concentration of the original distillate, together with the liquid remaining after the hydrolysis and distillation of the concentrated portion, were made alkaline with sodium hydroxide and evaporated to a small volume. The acids were then liberated by means of sulfuric acid and converted into barium salts in the manner previously described. As a considerable amount of formic acid was found to be present, this was first removed by oxidation with potassium permanganate, and by subsequent precipitation with silver nitrate the following fractions of silver salt were obtained, the first of which became slightly reduced on drying.

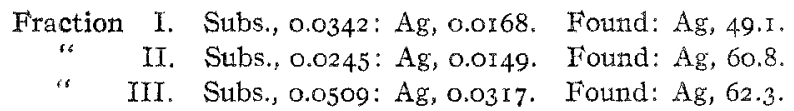

1 Compare Tschirch, Arch. Pharm., 252, 538 (I9r4); von Fellenberg, Biochem. $2 ., 85,45$ (I918).

${ }^{2}$ Lechartier and Bellamy, Compt. rend., 69, 356, 466 (1869); Pasteur, ibrd., 75, I054 (1872); Müntz, ivid., 86, 49 (1878); Berthelot, ibid., x28, x366 (I899).

${ }^{3}$ Ann., I 77, 344, 372, 382 (1875).

"Monatsh., 19, 333 (1898). Compare also Miller, Univ. Wisconsin, Bull. $99 \times$ ( 919 ).

5 Bull. soc. chim., 25, 433 (1876). 
These restllts indicated the acids to be chiefly formic and acetic, with a small amount of caproic acid.

On allowing the previously mentioned light petroleum extract to evaporate spontaneously in a suitable flask a very small amount of sub. stance was obtained which possessed a pleasant odor. This was oxidized with ro $\mathrm{cc}$. of a chromic acid mixture and further treated in the manner previously described. The amount of barium salt eventually obtained was too small for analysis, but a portion of it on the addition of dil. sulfuric acid gave a pronounced odor of valeric acid, and the remainder when heated with absolute alcohol and a few drops of sulfuric acid developed the characteristic odor of ethyl valerate. There could thus be no doubt respecting the occurrence of amyl alcohol or its esters in Springdale apples.

\section{Part III. Examination of Crab Apples.}

As crab apples possess a particularly fragrant odor, and were available to us in considerable quantities, it was deemed of interest to compare their odorous constituents with those of the previously examined Ben Davis apple. The variety employed for this purpose consisted of the cultivated yellow fruit known as the "Golden Beauty."

The operations of paring the apples and conducting the distillation were precisely the same as those described in the first part of this paper. The amount of parings employed was $77 \mathrm{~kg}$., which represented about $257 \mathrm{~kg}$. of the entire fruit, and the total amount of distillate collected was 76 liters.

The distillate, which had a fragrant, apple-like odor, was somewhat opalescent, but on keeping for a few days the liquid became clear and an oily film separated on the surface. Eiven the first portions of the distillate were perfectly neutral to test-paper. It seemed desirable in this investigation to compare the yield and characters of the essential oil with those of the analogous product obtained from Ben Davis apples, and the total amount of distillate was, therefore, cohobated in a current of steam until the odorous constituents were concentrated in a volume of ro liters. The first portions of this concentrated liquid were practically neutral and contained a considerable amount of acetaldehyde, but gave no reaction for furfural. On extracting the entire concentrated distillate with 4 successive portions of ether which was free from aldehyde, and removing the solvent, first by distillation and finally by spontaneous evaporation, there was obtained $3.3579 \mathrm{~g}$. of a pale yellow oil. This represented $0.0043 \%$ of the apple parings or about $0.0013 \%$ of the entire fruit.

The essential oil from crab apples possessed the same general character as that obtained from Ben Davis apples, and no material difference in odor could be observed, although the yield of oil from the former was somewhat larger. The presence of both furfural and acetaldehyde was established by special tests. 
After extracting the concentrated distillate with ether it was made alkaline with sodium hydroxide and distilled from a sand bath, the portion passing over below $97^{\circ}$ being collected. This distillate consisted chiefly of ether with a small aqueous layer. After separating the latter and washing the ether several times with water the aqueous liquids were united, saturated with common salt, extracted with light petroleum (b. p. $30-55^{\circ}$ ), and distilled. On concentrating this distillate, and testing in the previously described manner the small portion which was first collected, a decided reaction for methyl alcohol was obtained. As the ether originally exnployed for extracting the aqueous distillate, when similarly tested, gave a perfectly negative result, the presence of minute amounts of methyl alcohol or its ester in the crab apple was definitely determined.

The above-mentioned, aqueous, alkaline liquid, from which the portion boiling below $97^{\circ}$ had been removed, was acidified with sulfuric acid and distilled in a current of steam until the distillate was practically neutral. The volatile acids were subsequently converted into barium salts, and as the latter were found to contain a considerable amount of formic acid, this was removed in the previously described manner by means of potassium permanganate. After finally concentrating the neutral solution it yielded with silver nitrate the following fractions of silver salt, which were analyzed.

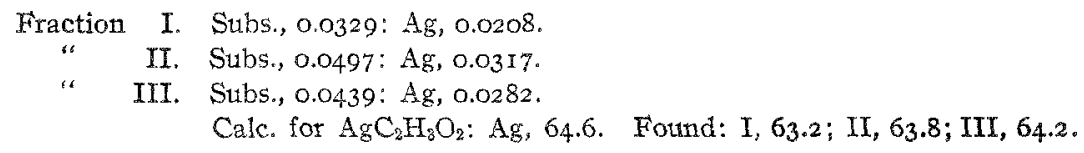

The concentrated distillate from crab apples, after extraction of the essential oil, was thus found to contain chiefly formic and acetic acids, with a very small proportion of an acid of higher molectlar weight.

Examination of the Aqueous Liquids Remaining from the Cohobation of the Original Distillate. Identification of Formic, Acetic, and Caproic Acids.

The large amount of nearly odorless liquid remaining from the cohobation of 76 liters of the original crab apple distillate was made alkaline with sodium hydroxide and evaporated to a comparatively small bulk. It was then acidified with sulfuric acid, and subjected to distillation in a current of steam. The first portion of the distillate was strongly acid, had an odor of the higher fatty acids, and separated an oily film on the surface. After converting the entire amount of volatile acid into a barium salt, the latter was found to contain a considerable proportion of formate, and this was, therefore, removed by treatment with potassium permanganate in the manner previously described. The concentrated, neutral solution eventually obtained yielded with silver nitrate several successive fractions of silver salt, which were collected, dried, and analyzed. 
Fraction I. Subs., 0.0205: Ag, 0.0099 .

"II. Subs, , 0.0284: Ag, 0.0138.

"III. Subs, 0.0439: Ag, 0.0215.

Calc. for $\mathrm{AgC}_{6} \mathrm{H}_{11} \mathrm{O}_{2}: \mathrm{Ag}, 48.4$. Found: I, 48.3; II, 48.6; III, 48.9.

All the above fractions evidently consisted of nearly pure silver caproate. By concentrating the filtrate and washings from the above-mentioned precipitates several additional fractions of silver salt were obtained.

\begin{tabular}{|c|c|c|c|}
\hline Fraction. & Subs. & $\begin{array}{l}\text { Ag. Found. } \\
\text { G. }\end{array}$ & $\%$ \\
\hline IV $\ldots$ & 0.0353 & 0.0178 & 50.4 \\
\hline $\mathrm{V}$ & 0.1212 & $0.06 r 6$ & 50.8 \\
\hline VI... & 0.0530 & 0.0273 & $5 T \cdot 5$ \\
\hline VII.... & 0.0634 & 0.0329 & 5 I. 9 \\
\hline $\operatorname{VIII} \ldots \ldots \ldots \ldots \ldots \ldots$ & 0.0805 & 0.0477 & $59 \cdot 3$ \\
\hline $\mathrm{IX} \ldots \ldots \ldots \ldots \ldots \ldots$ & 0.0724 & 0.0457 & $63 \cdot x$ \\
\hline $\mathbf{x} \ldots \ldots \ldots$ & 0.0764 & 0.0487 & $63 \cdot 7$ \\
\hline$x r \ldots \ldots \ldots \ldots \ldots$ & 0.0334 & 0.0215 & 64.4 \\
\hline
\end{tabular}

In view of the composition of the first 3 fractions, and the gradual increase in percentage of silver, it may be considered that Fractions IV to $\mathrm{X}$ consisted of mixtures of silver caproate and acetate, especially as the last fraction was evidently nearly pure acetate. The rather close agreement of Fractions VI and VII with si lver valerate, which requires 5 r.7\% $\mathrm{Ag}$, is regarded merely as a coincidence, for the odor developed by all the salts on ignition was similar to that produced by caproic acid, and throughout the investigation there was otherwise no evidence of the presence of valeric acid.

The above results have thus shown the volatile acids of the crab apple to be the same as those of the Ben Davis apple, consisting chiefly of formic, acetic, and caproic acids, and, as will be indicated later, a very small amount of caprylic acid is also undoubtedly present.

Separation of the Aldehyde from the Distillate of Crab Apples, and its Further Identification as Acetaldehyde.

In the preceding examination of the apple distillates the presence of a notable quantity of acetaldehyde was observed, but its identification was effected only by the described qualitative tests, which included, however, the characteristic reaction with dimethylamine and sodium nitroprusside. Inastuuch as the plan of the previously described investigation necessitated the treatment of the concentrated distillate with a caustic alkali, in order to effect the hydrolysis of the esters, and as this resulted in the destruction of the aldehyde, it was deemed desirable to conduct some special experiments whereby the identity of the aldehydic constituent could be further confirmed. It was also considered important to ascertain that the acetaldehyde was not accompanied by a higher homolog, since some of these 
compounds possess highly odorous properties, and incidentally the alcoholic constituent of the crab apple esters has also been determined.

For the above-mentioned purpose $17.24 \mathrm{~kg}$. of fresh crab apple parings were brought immediately into a still, and the material subjected to distillation in a current of steam. The first portion of the distillate, being concentrated, was set aside, and the distillation then continued so long as any odorous substances passed over. The weaker distillate was subsequently concentrated by repeated cohobation, until finally the concentrated distillate anounted to about $500 \mathrm{cc}$. This liquid was saturated with sodium hydrogen sulfite, and the mixture set aside for several days, after which it was extracted 5 times successively with pure, aldehyde-free ether. The examination of these ethereal liquids will subsequently be described. The sulfite solution was made alkaline with sodium carbonate and distilled in a current of steam until about $500 \mathrm{cc}$. of distillate was obtained. The first portion of the distillate possessed an odor which was chiefly that of acetaldehyde, but also reminding somewhat of a higher aldehyde, and the total distillate was slightly opalescent. It yielded in a high degree all the previously described aldehyde reactions, including that characteristic of acetaldehyde. A special test for formaldehyde with phenylhydrazine hydrochloride gave a perfectly negative result, thus confirming the previous observations regarding the absence of this compound in the apple distillate.

The distillate containing the aldehyde was treated with an alkaline solution of potassium permanganate until the red color was permanent, after which it was filtered, the excess of permanganate removed by the addition of a solution of ferrous sulfate in dil. sulfuric acid, and the mixture distilled in steam. The entire acid distillate, which had a very slight odor, was made alkaline with barium hydroxide, and the liquid filtered and concentrated. On the subsequent addition of a few drops of solution of silver nitrate a slight blackish precipitate was produced, which was removed by filtration, after which by fractional precipitation a series of perfectly white silver salts was obtained. These were dried over sulfuric acid and analyzed.

\begin{tabular}{|c|c|c|c|}
\hline Fraction. & Subs. & $\begin{array}{l}\text { Ag. Found. } \\
\text { G. }\end{array}$ & $\%$ \\
\hline I... & 0.0486 & $0.03 \mathrm{II}$ & 64.0 \\
\hline$\pi \ldots \ldots \ldots \ldots \ldots \ldots \ldots$ & 0.0374 & 0.0240 & 64.2 \\
\hline MI $\ldots \ldots \ldots \ldots \ldots \ldots \ldots$ & 0.0558 & 0.0359 & $64 \cdot 3$ \\
\hline $\mathbb{I V} \ldots \ldots \ldots \ldots \ldots \ldots$ & 0.0485 & 0.0312 & $64 \cdot 3$ \\
\hline$\forall \ldots \ldots \ldots \ldots \ldots \ldots$ & 0.0822 & 0.0527 & $64 . \mathrm{r}$ \\
\hline
\end{tabular}

These results indicate that the aldehyde obtained from the crab apple was nearly pure acetaldehyde, with possibly a trace of a higher homolog.

The total amount of silver salt obtained was $0.6493 \mathrm{~g}$., which would 
correspond to 0.17 II $g$. of acetaldehyde, or about $0.001 \%$ of the weight of apple parings employed. Inasmuch as the yield of essential oil, which naturally would contain but little of the aldehyde, was $0.0043 \%$, the proportion of the latter in the original apple distillate was relatively large.

\section{Hydrolysis of the Esters. Identification of Amyl Alcohol.}

As previously mentioned, the concentrated crab apple distillate after saturation with sodium hydrogen sulfite was extracted several times with ether in order to remove the non-aldehydic substances. The united ethereal liquids, which gave no reaction for aldehyde, were washed with a little water, dried with anhydrous sodium sulfate, and the greater part of the ether removed by distillation. The liquid was then brought into a small, tared flask and the remainder of the ether allowed to evaporate spontaneously, when there was obtained $0.2677 \mathrm{~g}$. of a yellowish oil which had an intense, and, when diluted, pleasant apple-like odor. This represented $0.0015 \%$ of the weight of apple parings. The oily product was mixed with $200 \mathrm{cc}$, of water, Io g. of sodium hydroxide added, and the mixture heated for 2 hours in a flask provided with an inverted condenser. It was then allowed to cool and distilled in a current of steam. The first portion of the distillate separated some oily drops and had the odor of any alcohol. The entire distillate was extracted 5 times with pure, aldehyde-free ether, the united ethereal liquids dried with anhydrous sodium sulfate, and the greater part of the ether removed by distillation, the remainder being then allowed to evaporate spontaneously in a flask provided with a glass stopper. To this residue, consisting of a few drops of yellowish oily liquid, to ce. of the previously mentioned chromic acid mixture was added, and the whole heated on a water bath, with occasional agitation, for about is minutes. It was then cooled, diluted with water, and extracted 5 times with pure ether. The united ethereal liquids were washed with a little water, and then extracted 4 times successively with a, $5 \%$ solution of barium hydroxide in portions of $25 \mathrm{cc}$. each. These alkaline liquids were saturated with carbon dioxide to remove the excess of baryta, and the filtered solution concentrated. On the subsequent addition of silver nitrate a very small amount of a tearly white precipitate was obtained, which was collected, washed with a little water and dried.

Subs, 0.0196: Ag, 0.0100.

Calc. for $\mathrm{AgC}_{5} \mathrm{H}_{3} \mathrm{O}_{2}: \mathrm{Ag}, 5$ r.7. Found: 5 r.0.

This result showed that the salt was silver valerate, and it afforded evidence that the crab apple, like ordinary apples, contains amyl alcohol in the form of esters.

No further amount of silver salt could be obtained from the abovementioned solution, but the evaporated mother-liquor on the addition 
of dil. sulfuric acid developed the odor of valeric acid. The ethereal liquid from which the valeric acid had been extracted by means of baryta was washed with water, dried with anhydrous sodium sulfate, and the ether removed, but it yielded a scarcely perceptible residue.

\section{Acids Obtained by the Hydrolysis of the Esters.}

The strongly alkaline liquid remaining after the hydrolysis of the esters and subsequent distillation was acidified with sulfuric acid and again distilled in a current of steam. The first portion of distillate was opalescent, and a very small amount of solid substance separated in the condenser, but it was gradually carried over and probably consisted of caprylic acid, which melts at $16.5^{\circ}$. The entire amount of volatile acid so obtained was converted into a barium salt, and the concentrated soltution of the latter yielded with silver nitrate a perfectly white silver salt, which was analyzed.

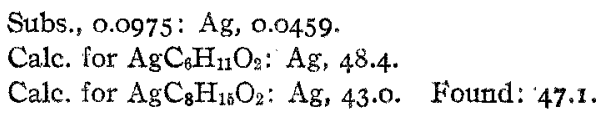

This result indicated the salt to consist of a mixture of silver caproate and caprylate, the former largely predominating.

\section{Acids Remaining in the Original Distillate after Cohobation.}

The large volume of aqueous liquid remaining from the concentration of the original distillate by cohobation was made alkaline with sodium hydroxide and evaporated. It was then acidified with sulfuric acid and distilled in a current of steam, the volatile acids being subsequently converted into a barium salt. As the solution of the latter was found to contain a considerable amount of formic acid, this was removed by means of potassium permanganate in the manner previously described. The filtered and concentrated neutral liquid eventually obtained gave with silver nitrate a small precipitate, which was collected, dried, and analyzed.

Fraction I. Subs., 0.0450: Ag, 0.0224. Found: Ag. 49.8 .

This salt evidently consisted chiefty of silver caproate, which requires $48.4 \% \mathrm{Ag}$, together with a little acetate. By the evaporation of the mother-liquor two other portions of salt were successively obtained and analyzed.

Fraction II. Subs, $0.0635: \mathrm{Ag}, 0.0384$.

III. Stubs., $0.05 \mathrm{I} 6: \mathrm{Ag}, 0.0328$.

Calc. for $\mathrm{AgC}_{2} \mathrm{H}_{3} \mathrm{O}_{2}: \mathrm{Ag}$, 64.6. Found: I, 60.5; II, 63.6.

From the above results it may be concluded that the aqueous liquid remaining after cohobation of the original distillate contained chiefly formic and acetic acids with a small proportion of caproic acid.

Part IV. Emanation of Acetaldehyde from Ripe Apples.

The occurrence of acetaldehyde among the products of distillation of odorous substances has frequently been observed in recent years, and it 
has indeed been stated ${ }^{1}$ that it is probably never entirely absent in any essential oil, although on account of its ready solubility in water, as well as its great volatility, it is mostly contained in the water of the distillate from which the oil has been separated. It has, however, hitherto been assumed that the lower aliphatic aldehydes are first produced in the process of distillation, and that they do not exist as such in the plant. ${ }^{2}$ Semmler ${ }^{3}$ has particulariy noted that from the standpoint of vegetable physiology acetaldehyde would gain in importance if it could be proved that it occurs ready formed in the plant. Inasmuch as the present authors had found that considerable amounts of acetaldehyde are contained in the distillate from ripe apples, it was deemed of special interest to ascertain whether this compound could be detected in the odorous emanation from the fruit, and the experiments in this direction have been completely successiul.

In the month of December a quantity of sound red apples of the variety known as "Nero" was brought into a large copper vessel, provided with a tightly fitting cover and a small opening both at the top and the bottom, so that a current of air could be slowly aspirated through it. In this experiment 215 apples were used, which weighed $31.85 \mathrm{~kg}$. The air which entered the vessel at the top was first passed through an alkaline solution of potassium permanganate and then through conc. sulfuric acid. At the bottom the exit tube was first connected with a small inclined glass apparatus consisting of a series of bulbs and containing a saturated solution of sodium hydrogen sulfite. To this bulb apparatus an empty gas washing bottle was attached and to the latter a similar bottle containing a saturated solution of sodium hydrogen sulfite, the whole being then connected through an intervening empty flask with a filter pump, by means of which the air was continuously drawn through the entire system. This operation was allowed to proceed uninterruptedly for 7 or 8 days, when the absorbing apparatus was detached, and the liquid through which the air had passed was then found to possess a pleasant apple-like odor. The entire amount of sulfite solution contained in the bulb apparatus and gas washing bottle was about $300 \mathrm{cc}$. It was made alkaline with sodium carbonate and distilled, the first distillate of about $50 \mathrm{cc}$. being then again distilled and $5 \mathrm{cc}$. of liquid collected, the receptacle having been cooled with ice. The concentrated distillate so obtained had the characteristic odor of acetaldehyde, inmediately reduced an ammoniacal solution of silver oxide in the cold, and gave within a few seconds a deep pink coloration with Schiff's reagent. It also gave with dimethyl-

${ }^{3}$ Gildemeister and Hoffmann, "Die aetherischen Oele," second edition, Bd. II, p. 480.

I Tbid, Bd. T, p. 422

"Senmler, "Die aetherischen Oese," Bd. r, p. 568, rgot. 
amine $e^{1}$ and sodium nitroprusside the deep indigo-blue color which is characteristic of acetaldehyde.

The above-described operation was subsequently conducted with several other varieties of apples, which were loosely packed in a large conical glass percolator, but otherwise the conditions were the same.

The second experiment was made early in January, and 60 "Paragon" apples were used, which weighed $9.37 \mathrm{~kg}$. For the third experiment, begun in the middle of January, a green variety of apple known as the "Keeper" was employed, and 57 of them weighed 9.18 kg. A final experiment was made early in February with crab apples, which were of the same kind and quality as those used for the previously described chemical examination of this fruit. This variety of apple being small in size the number used was 150 , which weighed $9.6 \mathrm{~kg}$.

In all the above-mentioned experiments the aldehyde was liberated from its bisulfite compound, and in the subsequent tests for its identification the same decisive results were always obtained. The interesting fact has thus been established that the odorous emanations from the apple during its period of vital activity contain acetaldehyde, and it follows that the occurrence of this compound in a distillate from the fruit does not depend upon any chemical changes in the process of distillation.

\section{Summary.}

The results of the present investigation, for which several varieties of apples were employed, may be briefly summarized as follows.

I. The odorous constituents of apples have been found to consist essentially of the amyl esters of formic, acetic, and caproic acids, with a very small amount of the caprylic ester and a considerable proportion of acetaldehyde. In considering the relative amounts of the respective alcohol and acids it seems probable that the latter are also present to some extent in the free state.

2. It has been shown that acetaldehyde is a product of the vital activities of the fruit, and that it occurs in the exhalations of ripe apples. This observation is deemed of considerable biological importance, especially as it has hitherto been assumed that the lower aliphatic aldehydes are first formed during the process of steam distillation. It is, for example, quite probable that the exhalation of acetaldehyde may prove to be one of the factors involved in the production of so-called "apple-scald," an affection to which some apples are subject when stored without free circulation of air. ${ }^{2}$

3. The aqueous distillate from fresh apple parings has been found to

1 As several works of reference designate the 11se of trimethylamine in the specific test for acetaldehyde, the fact may again be noted that the reaction is not obtained with this reagent, unless it may by chance contain some dimethylatnine.

"Compare Brooks, Cooley and Fisher, J. Agr. Res., I8, $211-240$ (xgrg). 
contain, in addition to the above-mentioned substances, exceedingly small amounts of methyl and ethyl alcohols and also a small amount of furfural. Inasmuch as the last compound is doubtless produced by chemical changes in the material during its distillation, it can not be regarded as one of the odorous constituents of the apple.

4. The essential oil, as extracted by means of ether from a concentrated distillate of either ordinary apple parings or those of the crab apple, is at ordinaty temperatures a yellowish, somewhat viscid liquid, becoming much darker on keeping. When slightly cooled it forms a concrete mass, due to the separation of small acicular crystals, which consist of a paraffin hydrocarbon. It possesses in a high degree the characteristic, fragrant odor of fresh apples. Besides the esters mentioned, it has been found to contain, by specific tests, small amounts of acetaldehyde and furfural. The yield of oil from the parings of the Ben Davis apple was $0.0035 \%$, and that from the more odorous crab apple $0.0043 \%$, which corresponds to about 0.0007 and $0.0013 \%$ respectively of the entire ripe fruit.

5. Although amyl valerate is generally designated in chemical literature as "apple oil," it is quite certain that this compound has never been identified as a constituent of apples, and in the course of this investigation no evidence could be obtained of its presence. On the other hand it has been shown that the characteristic, fragrant odor of ripe apples is due to a mixture of the previously enumerated substances, which may exist in varying proportions in the numerous varieties of the fruit, thus giving rise to slight differences of odor.

WASAISTGON, D. C.

\section{NEW BOOKS.}

Practical Physiological Chemistry. A Book Designed for Use in Courses in Practical Physiological Chemistry in Schools of Medicine and of Science. 6th edition, revised and enlarged. By PHIL.TP B. HAwk, M.S., PH.D., Prof. of Physiological Chemistry and Toxicology in the Jefferson Medical College of Philadelphia. P. Blakiston's Son \& Co., Philadelphia, I. 9 I8. xiv +661 pp., 185 figs. and 6 plates. $16 \times 24 \mathrm{~cm}$. $\$ 3.50$ net.

Despite the fact that the general plan of this book was adversely criticized when it first appeared more than ro years ago, it has probably found more widespread use as a laboratory manual and text of physiological chemistry than any other book. The reason for this is not hard to find. Although this book contains more exercises and quantitative methods than it would be possible to utilize in an extended course in physiological chemistry, it allows the instructor such a wide choice in the selection of the exercises he might desire to employ as to fit almost any need. As a handbook of quantitative methods in physiological and pathological chemistry the book is most valuable, since nearly all the methods which the laboratory worker has occasion to use may be found in this handy 